\title{
THE INVESTIGATION OF GHOST FLUID METHOD FOR SIMULATING THE COMPRESSIBLE TWO-MEDIUM FLOW
}

\author{
HAI TIAN LU \\ College of Aerospace Engineering, Nanjing University of Aeronautics and Astronautics \\ Nanjing, 210016, the People's Republic of China \\ lhtgkzy@126.com \\ NING ZHAO* \\ College of Aerospace Engineering, Nanjing University of Aeronautics and Astronautics \\ Nanjing, 210016, the People's Republic of China \\ nzhao2000@hotmail.com \\ DONGHONG WANG \\ College of Science, Nanjing University of Aeronautics and Astronautics \\ Nanjing, 210016, the People's Republic of China \\ wangdonghong@nuaa.edu.cn
}

Published 22 June 2016

\begin{abstract}
In this paper, we investigate the conservation error of the two-dimensional compressible twomedium flow simulated by the front tracking method. As the improved versions of the original ghost fluid method, the modified ghost fluid method and the real ghost fluid method are selected to define the interface boundary conditions, respectively, to show different effects on the conservation error. A Riemann problem is constructed along the normal direction of the interface in the front tracking method, with the goal of obtaining an efficient procedure to track the explicit sharp interface precisely. The corresponding Riemann solutions are also used directly in these improved ghost fluid methods. Extensive numerical examples including the sod tube and the shock-bubble interaction are tested to calculate the conservation error. It is found that these two ghost fluid methods have distinctive performances for different initial conditions of the flow field, and the related conclusions are made to suggest the best choice for the combination.
\end{abstract}

Keywords: Conservation error; front tracking method; modified ghost fluid method; real ghost fluid method; two-medium flow.

\section{Introduction}

The investigation of interfaces separating multi-medium flows is of significant realistic value in many scientific fields and engineering applications. The interfacial instability phenomenon in the fluid dynamics, for instance, the Richtmyer-Meshkov (R-M)

${ }^{*}$ Corresponding author. College of Aerospace Engineering, Nanjing University of Aeronautics and Astronautics, Nanjing, 210016, the People's Republic of China, nzhao2000@hotmail.com.

This is an Open Access article published by World Scientific Publishing Company. It is distributed under the terms of the Creative Commons Attribution 3.0 (CC-BY) License. Further distribution of this work is permitted, provided the original work is properly cited. 
instability $^{1}$ and Richtmyer-Taylor (R-T) instability ${ }^{2}$ are the important research in the inertial confinement fusion and have great influences on the implosion and combustion of fusion materials.

Accurate simulations of multi-medium flows with sharp front present a problem of considerable difficulty, which are mainly consisted of two parts: one is to solve the single medium flow precisely and the other is to treat the material interface accurately. On the one hand, in recent twenty years, the discontinuous Galerkin (DG) method performed very well in the simulations of single medium compressible flow. The original DG method was introduced by Reed and $\mathrm{Hill}^{3}$ for solving the linear equations in the framework of neutron transport. A major development of the DG method was carried out by Cockburn and Shu in a series of papers ${ }^{4-8}$. They employed the total variation diminishing (TVD) high order Runge-Kutta time discretizations ${ }^{9}$ and DG discretization in space with exact or approximate Riemann solvers as interface fluxes and total variation bounded (TVB) limiter ${ }^{10}$ to achieve non-oscillatory property for strong shocks. These schemes are termed Runge-Kutta discontinuous Galerkin (RKDG) method. On the other hand, a relatively dominant part for simulating compressible two-medium flow is the treatment of the moving material interface and its vicinity. The earlier algorithms which have treated the material interface by the $\gamma$-based mode ${ }^{11}$, the mass-fraction mode ${ }^{12-13}$ or a level set function ${ }^{14-15}$, usually yield a numerical diffusion of contact discontinuities over several nodes. However, for the front tracking method ${ }^{16-19}$, fluid interfaces are explicitly tracked by connected marker points and a sharp interface boundary is maintained during the computation. With this method, accurate boundary conditions can be imposed at moving interfaces and non-physical compression can be relatively easy to avoid for the incompressible flow.

It is worth mentioning that the ghost fluid method (GFM) presents a robust and efficient way to treat multi-medium flows in the definition of interface boundary conditions. The original GFM is introduced by Fedkiw ${ }^{20-22}$, where the material interface is "invisible" by defining the ghost fluids and thus the computations are carried out as if it is in the single medium. However, when the pressure or the velocity experiences a large gradient across the interface, the original GFM may cause numerical inaccuracy. Indeed, the ghost fluid states should consider the influence of both wave interaction and material properties on the interfacial evolution. This leads to the proposal of improved versions of GFM, for example, the modified ghost fluid method ${ }^{23-25}$ (MGFM) and the real ghost fluid method $^{26}$ (RGFM). In the MGFM, a Riemann problem is defined and solved approximately to predict interface states which are then used to define the ghost fluid states. However, only the density in the real fluid nodes adjacent to the interface is modified isentropically to suppress the possible overheating problem. The RGFM further predicts the density, normal velocity and pressure for the real fluid nodes just next to the interface by the corresponding Riemann solutions because wave interaction at the interface can propagate upward and downward simultaneously. The RGFM enables a better imposition of interface boundary conditions, especially for those critical problems of shock impendence matching. To summarize, the main distinction between MGFM and 
RGFM lies in the implementation with the real fluid states near the interface, which then leads to different performances for various compressible two-medium flow problems. Therefore it is necessary to compare and make conclusions to provide the best choice of the GFMs (i.e., MGFM and RGFM) according to the specific initial conditions of flow field.

In this paper, our major intention is to investigate the different effects with GFMs to solve for the compressible two-medium flow in two dimensions. Since there is nearly no analytical solution in two dimensions, the conservation error is measured to act as an indicator. The interface motion in the front tracking method is determined by the Riemann problem constructed along the normal direction of the interface and the method in Ref. 18 is used to reconstruct the interface. The flow field is computed by the RKDG method in single medium and the main purpose to use RKDG method is in the following: Firstly, the higher order accuracy is obtained in smooth regions easily. Secondly, the input states to the Riemann problem constructed near the interface are obtained from solution polynomials directly in contrast to the interpolation used in the finite difference method. Finally, the good compactness requires less ghost fluid information (surface normal vector, ghost fluid states, etc) since these values far from the interface are less accuracy.

The organization of this paper is as follows: in Section 2 and Section 3, we review and use the RKDG method with front tracking method for solving the two-medium flow and describe the implementation with GFMs in detail. Extensive numerical tests are presented in Section 4 to compare the conservation error caused by the MGFM and the RGFM, respectively. Concluding remarks are then given in Section 5.

\section{Equations and numerical method}

\subsection{Governing equation}

We consider the two-dimensional Euler equation

$$
\frac{\partial \vec{U}}{\partial t}+\nabla \cdot \vec{F}(\vec{U})=0
$$

where $\vec{U}=[\rho, \rho u, \rho v, E]^{T}, \vec{F}(\vec{U})=\left[\vec{F}_{1}(\vec{U}), \vec{F}_{2}(\vec{U})\right], \vec{F}_{1}(\vec{U})=\left[\rho u, \rho u^{2}+p, \rho u v,(E+p) u\right]^{T}$, $\vec{F}_{2}(\vec{U})=\left[\rho v, \rho u v, \rho v^{2}+p,(E+p) v\right]^{T}$. Here $\rho$ is the density, $u$ and $v$ are the velocities, $p$ is the pressure, $E$ is the total energy per unit volume. The total energy is given as

$$
E=\rho e+\rho\left(u^{2}+v^{2}\right) / 2,
$$

where $e$ is the internal energy per unit mass. The stiffened gas equation of $\operatorname{state}^{27}$ is used

$$
p=(\gamma-1) \rho e-\gamma B
$$

where $\gamma$ and $B$ are treated as fluid constants, and will be specified in Section 4 . 


\subsection{The implementation of the RKDG method}

Here we briefly describe the two-dimensional RKDG method. Consider the rectangular cell $I_{i, j}=\left[x_{i-1 / 2}, x_{i+1 / 2}\right] \times\left[y_{j-1 / 2}, y_{j+1 / 2}\right]$ with the cell center $\left(x_{i}, y_{j}\right)$, we denote the rectangular cell size by $\Delta x=x_{i+1 / 2}-x_{i-1 / 2}$ and $\Delta y=y_{j+1 / 2}-y_{j-1 / 2}$. Let $h=\max (\Delta x, \Delta y)$ and replace the exact solution $\vec{U}$ by the numerical solution $\vec{U}^{h}$, the numerical solution as well as the test function space is given by $V_{h}^{k}=\left\{p:\left.p\right|_{I_{i, j}} \in P^{k}\left(I_{i, j}\right)\right\}$, where $P^{k}\left(I_{i, j}\right)$ is the space of polynomials with degree no greater than $k$ on the cell $I_{i, j}$. The local orthogonal basis functions over $I_{i, j}$ are adopted as

$$
\begin{aligned}
& \phi_{0}(x, y)=1, \\
& \phi_{1}(x, y)=\frac{x-x_{i}}{\Delta x / 2}, \\
& \phi_{2}(x, y)=\frac{y-y_{j}}{\Delta y / 2}, \\
& \phi_{3}(x, y)=\phi_{1}(x, y) \phi_{2}(x, y), \\
& \phi_{4}(x, y)=\phi_{1}^{2}(x, y)-\frac{1}{3}, \\
& \phi_{5}(x, y)=\phi_{2}^{2}(x, y)-\frac{1}{3},
\end{aligned}
$$

The numerical solution $\vec{U}^{h}(x, y, t)$ can then be written in the test function space $V_{h}^{k}$ as

$$
\vec{U}^{h}(x, y, t)=\sum_{l=0}^{L} \vec{U}_{i, j}^{(l)}(t) \phi_{l}(x, y), \quad \text { for }(x, y) \in I_{i, j},
$$

and the degrees of freedom $\vec{U}_{i, j}^{(l)}(t)$ are the moments defined by

$$
\vec{U}_{i, j}^{(l)}(t)=\frac{1}{a_{l}} \int_{I_{i, j}} \vec{U}^{h}(x, y, t) \phi_{l}(x, y) d x d y, \quad l=0,1, \cdots, L,
$$

where $a_{l}=\int_{I_{i, j}}\left(\phi_{l}(x, y)\right)^{2} d x d y$ are the normalization constants. By substituting Eq. (5) into Eq. (1), and multiplying Eq. (1) with a test function $\phi_{l}(x, y)$, integrating over the cell $I_{i, j}$ by parts, we can obtain the governing equations for the moments as

$$
\begin{aligned}
& \frac{d \vec{U}_{i, j}^{(l)}(t)}{d t}=\frac{1}{a_{l}}\left(-\sum_{e \in \partial I_{i, j}} \int_{e} \vec{F}\left(\vec{U}^{h}(x, y, t)\right) \cdot n_{e, I_{i, j}} \phi_{l}(x, y) d \Gamma\right. \\
& \left.+\int_{I_{i, j}} \vec{F}\left(\vec{U}^{h}(x, y, t)\right) \cdot \operatorname{grad} \phi_{l}(x, y) d x d y\right), \quad l=0,1, \cdots, L,
\end{aligned}
$$

where $n_{e, I_{i, j}}$ is the outward unit normal to edge $e$ of cell $I_{i, j}$. The integrals are approximated by a Gaussian quadrature formula with sufficient accuracy as 


$$
\begin{gathered}
\int_{e} \vec{F}\left(\vec{U}^{h}(x, y, t)\right) \bullet n_{e, I_{i, j}} \phi_{l}(x, y) d \Gamma \approx \sum_{s=1}^{S} \omega_{s} \vec{F}\left(\vec{U}^{h}\left(x_{e s}, y_{e s}, t\right)\right) \bullet n_{e, I_{i, j}} \phi_{l}\left(x_{e s}, y_{e s}\right)|e| \\
\int_{I_{i, j}} \vec{F}\left(\vec{U}^{h}(x, y, t)\right) \cdot \operatorname{grad} \phi_{l}(x, y) d x d y \approx \sum_{m=1}^{M} \bar{\omega}_{m} \vec{F}\left(\vec{U}^{h}\left(x_{I_{i, j} m}, y_{I_{i, j} m}, t\right)\right) \cdot \operatorname{grad} \phi_{l}\left(x_{I_{i, j} m}, y_{I_{i, j} m}\right)\left|I_{i, j}\right| \cdot(9)
\end{gathered}
$$

See Refs. 4 and 8 for more details of the numerical quadrature formulas. The flux $\vec{F}\left(\vec{U}^{h}\left(x_{e s}, y_{e s}, t\right)\right) \bullet n_{e, I_{i, j}}$ in the boundary integral is replaced by a monotone numerical flux. In this paper, the simple Lax-Friedrichs flux is used

$$
\begin{aligned}
& \vec{F}\left(\vec{U}^{h}\left(x_{e s}, y_{e s}, t\right)\right) \bullet n_{e, I_{i, j}} \approx \frac{1}{2}\left[\left(\vec{F}\left(\vec{U}^{+}\left(x_{e s}, y_{e s}, t\right)\right)+\vec{F}\left(\vec{U}^{-}\left(x_{e s}, y_{e s}, t\right)\right)\right) \bullet n_{e, I_{i, j}}-\right. \\
& \left.\alpha\left(\vec{U}^{+}\left(x_{e s}, y_{e s}, t\right)-\vec{U}^{-}\left(x_{e s}, y_{e s}, t\right)\right)\right]
\end{aligned}
$$

where $\alpha$ is the biggest absolute value of the eigenvalues of the Jacobian matrix in the $n_{e, I_{i, j}}$ direction, and $\vec{U}^{ \pm}\left(x_{e s}, y_{e s}, t\right)$ refers to the value of $\vec{U}^{h}$ in the neighboring cell and in the current cell $I_{i, j}$, respectively.

The semi-discrete scheme Eq. (7) can be generalized as

$$
\vec{U}_{t}=L(\vec{U})
$$

which is discretized in time by a third-order TVD Runge-Kutta discretizations ${ }^{9}$

$$
\begin{aligned}
& \vec{U}^{(1)}=\vec{U}^{n}+\Delta t L\left(\vec{U}^{n}\right), \\
& \vec{U}^{(2)}=\frac{3}{4} \vec{U}^{n}+\frac{1}{4} \vec{U}^{(1)}+\frac{1}{4} \Delta t L\left(\vec{U}^{(1)}\right), \\
& \vec{U}^{n+1}=\frac{1}{3} \vec{U}^{n}+\frac{2}{3} \vec{U}^{(2)}+\frac{2}{3} \Delta t L\left(\vec{U}^{(2)}\right) .
\end{aligned}
$$

If there are strong discontinuities in solution, oscillations and nonlinear instability may occur. Thus, a local slope limiter is used to overcome such difficulty. For a scalar equation, we denote

$$
\begin{array}{ll}
\vec{U}_{i+1 / 2, j}^{-}=\vec{U}_{i, j}^{(0)}(t)+\bar{U}_{i, j}^{x}, & \vec{U}_{i-1 / 2, j}^{+}=\vec{U}_{i, j}^{(0)}(t)-\overline{\bar{U}}_{i, j}^{x}, \\
\vec{U}_{i, j+1 / 2}^{-}=\vec{U}_{i, j}^{(0)}(t)+\bar{U}_{i, j}^{y}, & \vec{U}_{i, j-1 / 2}^{+}=\vec{U}_{i, j}^{(0)}(t)-\overline{\bar{U}}_{i, j}^{y},
\end{array}
$$

where

$$
\begin{array}{ll}
\bar{U}_{i, j}^{x}=\sum_{l=1}^{L} \vec{U}_{i, j}^{(l)}(t) \phi_{l}\left(x_{i+1 / 2}, y_{j}\right), & \bar{U}_{i, j}^{x}=-\sum_{l=1}^{L} \vec{U}_{i, j}^{(l)}(t) \phi_{l}\left(x_{i-1 / 2}, y_{j}\right), \\
\bar{U}_{i, j}^{y}=\sum_{l=1}^{L} \vec{U}_{i, j}^{(l)}(t) \phi_{l}\left(x_{i}, y_{j+1 / 2}\right), & \bar{U}_{i, j}^{y}=-\sum_{l=1}^{L} \vec{U}_{i, j}^{(l)}(t) \phi_{l}\left(x_{i}, y_{j-1 / 2}\right) .
\end{array}
$$

Then, we modify $\bar{U}_{i, j}^{x}, \overline{\bar{U}}_{i, j}^{x}, \bar{U}_{i, j}^{y}, \overline{\bar{U}}_{i, j}^{y}$ by 


$$
\begin{aligned}
& \bar{U}_{i, j}^{x(\bmod )}=\bar{m}\left(\bar{U}_{i, j}^{x}, \vec{U}_{i+1, j}^{(0)}(t)-\vec{U}_{i, j}^{(0)}(t), \vec{U}_{i, j}^{(0)}(t)-\vec{U}_{i-1, j}^{(0)}(t)\right), \\
& \overline{\bar{U}}_{i, j}^{x(\bmod )}=\bar{m}\left(\bar{U}_{i, j}^{x}, \vec{U}_{i+1, j}^{(0)}(t)-\vec{U}_{i, j}^{(0)}(t), \vec{U}_{i, j}^{(0)}(t)-\vec{U}_{i-1, j}^{(0)}(t)\right), \\
& \bar{U}_{i, j}^{y(\bmod )}=\bar{m}\left(\bar{U}_{i, j}^{y}, \vec{U}_{i, j+1}^{(0)}(t)-\vec{U}_{i, j}^{(0)}(t), \vec{U}_{i, j}^{(0)}(t)-\vec{U}_{i, j-1}^{(0)}(t)\right), \\
& \overline{\bar{U}}_{i, j}^{y(\bmod )}=\bar{m}\left(\bar{U}_{i, j}^{y}, \vec{U}_{i, j+1}^{(0)}(t)-\vec{U}_{i, j}^{(0)}(t), \vec{U}_{i, j}^{(0)}(t)-\vec{U}_{i, j-1}^{(0)}(t)\right),
\end{aligned}
$$

where $\bar{m}$ is a modified minmod function

$$
\bar{m}\left(a_{1}, \cdots, a_{m}\right)= \begin{cases}a_{1}, & \text { if }\left|a_{1}\right| \leq M \Delta x^{2}, \\ s \bullet \min _{i}\left|a_{i}\right|, & \text { if } s=\operatorname{sign}\left(a_{1}\right)=\cdots=\operatorname{sign}\left(a_{m}\right), \\ 0, & \text { otherwise, }\end{cases}
$$

where $M$ is the TVB limiter constant. For $\bar{U}_{i, j}^{y(\text { mod) }}$ and $\bar{U}_{i, j}^{y(\bmod )}, \Delta x$ is changed into $\Delta y$.

For the system cases, the limiter is always used with a local characteristic field decomposition. See Refs. 5 and 8 for details.

\section{The interface treatment}

\subsection{Tracking fluid interface}

As indicated in Fig. 1, the medium 1 and medium 2 are separated by the interface at time $t^{n}$. The marker points, represented by the black triangle, are intersections of the interface with grid lines. $\vec{N}_{P}=\left(N_{P x}, N_{P y}\right)$ is the unit normal vector of marker point $P\left(x_{P}, y_{P}\right)$ and its orientation is always from medium 1 to medium 2. Point $A\left(x_{A}, y_{A}\right)$ and point $B\left(x_{B}, y_{B}\right)$ are in different mediums, obtained by the distance $\Delta n$ from marker point $P$. Here

$$
\begin{gathered}
\Delta n=\alpha\left[\left(\frac{N_{x}}{\Delta x}\right)^{2}+\left(\frac{N_{y}}{\Delta y}\right)^{2}\right]^{-\frac{1}{2}}, \\
x_{A}=x_{P}+\Delta n \bullet N_{P x}, y_{A}=y_{P}+\Delta n \bullet N_{P y}, \\
x_{B}=x_{P}-\Delta n \bullet N_{P x}, y_{B}=y_{P}-\Delta n \bullet N_{P y},
\end{gathered}
$$

where $\alpha$ is a constant and is suggested to be $1 \sim 2$. The state vectors at point $A$ and point $B$ can be obtained directly from the solution polynomials in the RKDG method

$$
\begin{aligned}
\vec{U}_{A} & =\sum_{l=0}^{L} \vec{U}_{K_{A}}^{(l)}(t) \phi_{l}\left(x_{A}, y_{A}\right), \\
\vec{U}_{B} & =\sum_{l=0}^{L} \vec{U}_{K_{B}}^{(l)}(t) \phi_{l}\left(x_{B}, y_{B}\right),
\end{aligned}
$$


where $K_{A}$ and $K_{B}$ are the grid cells containing point $A$ and point $B$. The density, the normal velocity and the pressure are calculated, denoted by $\vec{W}_{A}=\left[\rho^{A}, u_{N}^{A}, p^{A}\right]^{T}$ and $\vec{W}_{B}=\left[\rho^{B}, u_{N}^{B}, p^{B}\right]^{T}$. Then a Riemann problem is established at the marker point $P$

$$
\vec{W}_{P}=\left\{\begin{array}{l}
\vec{W}_{B} \\
\vec{W}_{A}
\end{array} .\right.
$$

An approximate Riemann problem solver (ARPS) based on a doubled shock structure can be employed to obtain the Riemann solutions ${ }^{28}$. In this way, we get the Riemann solutions at the marker point $P$ and denote it as $\vec{W}_{P}=\left[\rho_{L}^{I}, \rho_{R}^{I}, u_{N}^{I}, p^{I}\right]^{T}$. As the tangential velocity of the marker point $P$ is not determined accurately from the Riemann problem, the method ${ }^{29}$ proposed by Cocchi et al. is used

$$
v_{T}^{I}= \begin{cases}v_{T}^{B}, & \text { if } u_{N}^{I} \geq 0, \\ v_{T}^{A}, & \text { otherwise, }\end{cases}
$$

where $u_{N}^{I}$ and $v_{T}^{I}$ are the normal and tangential velocities of marker point $P, v_{T}^{A}$ and $v_{T}^{B}$ are the tangential velocities of the point $A$ and point $B$.

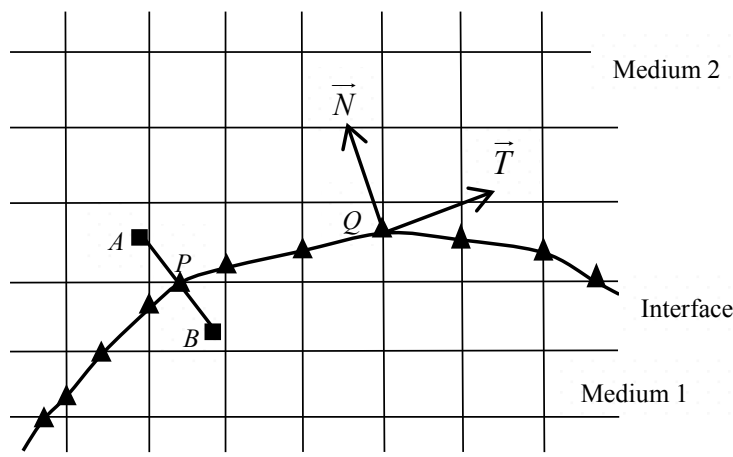

Fig. 1. Construction of Riemann problem at marker point.

Once the velocity of each marker point has been found, its new position is updated simultaneously with the governing equation by the same time integration scheme

$$
\begin{aligned}
& \vec{x}_{f}^{(1)}=\vec{x}_{f}^{n}+\Delta t \cdot \vec{v}_{f}\left(\vec{x}_{f}^{n}\right), \\
& \vec{x}_{f}^{(2)}=\frac{3}{4} \vec{x}_{f}^{n}+\frac{1}{4} \vec{x}_{f}^{(1)}+\frac{1}{4} \Delta t \bullet \vec{v}_{f}\left(\vec{x}_{f}^{(1)}\right), \\
& \vec{x}_{f}^{n+1}=\frac{1}{3} \vec{x}_{f}^{n}+\frac{2}{3} \vec{x}_{f}^{(2)}+\frac{2}{3} \Delta t \cdot \vec{v}_{f}\left(\vec{x}_{f}^{(2)}\right),
\end{aligned}
$$

where $\vec{x}_{f}^{n}$ and $\vec{x}_{f}^{n+1}$ are the positions of the marker points at time $t^{n}$ and $t^{n+1}$, respectively. $\vec{v}_{f}$ is the interface velocity vector, and $\Delta t$ is the time step. 


\subsection{MGFM and RGFM}

In the MGFM $^{24}$ and RGFM $^{26}$, a Riemann problem is defined at the interface and the ghost fluid states are obtained by solving an advection equation. However, since the Riemann problem has been constructed when tracking fluid interface, the Riemann solutions can be used directly. As indicated in Fig. 2, the medium 1 and medium 2 are separated by the interface. Points $R, S, P$ and $Q$ are the marker points near the cell $A . \vec{N}_{P}$ is the normal vector of marker point $P$ and $\vec{N}_{A}$ is the surface normal vector of the cell $A$. The fluid states at the cell $A$ can be updated by the marker point nearby. The marker point $P$ is selected if the angle between $\vec{N}_{P}$ and $\vec{N}_{A}$ is the minimum compared with other marker points. Assuming the Riemann solutions at the marker point $P$ are denoted as $\vec{W}_{P}=\left[\rho_{L}^{I}, \rho_{R}^{I}, u_{N}^{I}, p^{I}\right]^{T}$ according to the implementation in Section 3.1.

In the MGFM, only the density in the cell $A$ is updated isentropically to suppress the possible overheating problem

$$
\frac{p_{A}+B_{1}}{\rho_{A}^{\gamma_{1}}}=\frac{p^{I}+B_{1}}{\left(\rho_{L}^{I}\right)^{\gamma_{1}}},
$$

where $p_{A}$ is the real pressure in cell $A, \gamma_{1}$ and $B_{1}$ is the characteristic parameter of material in Eq. (3) for medium 1, $\rho_{A}$ is the updated density in the cell $A$. However, for the RGFM, the density, normal velocity and pressure are all updated by $\left(\rho_{L}^{I}, u_{N}^{I}, p^{I}\right)$ while the tangential velocity remains constant. The updated fluid states in cell $A$ are then projected to the base function in cell $A$, to obtain the zero-order moments. Since in general the derivatives of physical solutions are discontinuous across the interface, the higher-order moments are set to be zero. It is similar to update the fluid states in other real fluid cells adjacent to the interface.

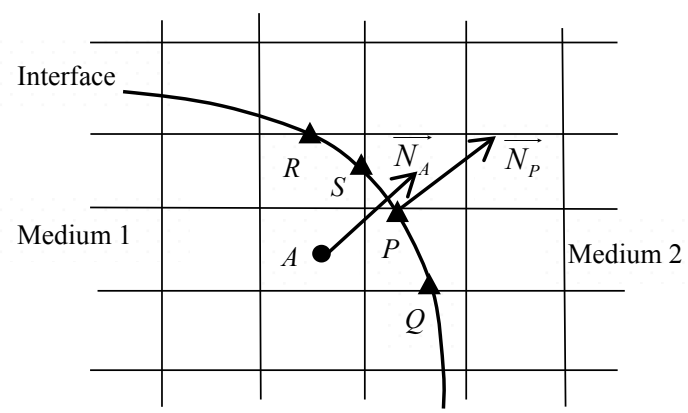

Fig. 2. Updating the real fluid states next to the interface by the Riemann solutions.

The ghost fluid states are obtained by solving the advection equation for both the MGFM and the RGFM

$$
\frac{\partial \phi}{\partial t} \pm \vec{N} \cdot \nabla \phi=0
$$


where $\phi$ represents the density, the normal velocity, the tangential velocity and the pressure in the ghost fluid region, $\vec{N}$ is the unit surface normal vector on ghost fluid cells. As indicated in Fig. 2, the "+" sign is used in Eq. (24) if the interface boundary conditions of medium 1 is to determine and the "-" sign is used in Eq. (24) if the interface boundary conditions of medium 2 is to determine. This advection equation is solved by iterating at each time step. Consider the compactness of the RKDG method, we only take 2-4 grid cells across the interface as the ghost fluid region. We then project the fluid states to the base function space in the ghost fluid cells to obtain the zero-order moments and set all the higher-order moments to be zero, and the interface boundary conditions is obtained.

\section{Numerical results}

In this section, the sod tube and shock-bubble interaction are tested under a series of initial conditions of flow field to measure the conservation errors caused by the GFMs, respectively. The compressible Euler equations are solved on uniform Cartesian grids with $P^{2}$ (third-order accurate) RKDG method. We take CFL number as 0.18 .

\subsection{Sod tube}

This is an example for a medium with higher energy expanding in other medium. Initially, the medium 1 is at the center of the computational domain $[0,2] \times[0,2]$ with a diameter of 1 . The nonreflecting boundary condition is used at all the boundaries and the TVB limiter constant is $M=0.4$. The computational domain is divided into $200 \times 200$ mesh cells. The interface is expanding outwards and the numerical solutions should be symmetrical. The initial conditions are

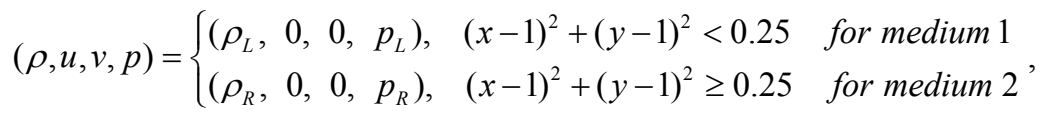

where $\rho_{L}, \rho_{R}, p_{L}, p_{R}$ are the density and pressure for medium 1 and medium 2, respectively. The conservation error $M_{C}$ is measured here

$$
M_{C}=\left(M_{n}-M_{0}\right) / M_{0},
$$

where $M_{n}$ is the flow field mass at time $t^{n}$ and $M_{0}$ is the initial flow field mass. Since the GFMs are implemented by the Riemann solutions at the interface and the constant $\alpha$ in Eq. (17) determines the input states of the Riemann problem, here we set $\alpha=1,2$ to consider this effect to the conservation error.

\subsubsection{Gas-gas sod tube}

We first investigate the case where both the medium 1 and medium 2 are ideal air $(\gamma=1.4$, $B=0)$. Keep $\rho_{R}$ and $p_{R}$ as constant by: $\rho_{R}=0.125, p_{R}=0.1, \rho_{L}$ and $p_{L}$ are increased by setting the density ratio and pressure ratio as: $\rho_{L} / \rho_{R}=24,40,56,72,88,104,120,136,152,168$, $184,200, p_{L} / p_{R}=10,100,1000,10000$. Fig. 3 gives the conservation error under a series 
of density ratio and pressure ratio. According to the sign of the conservation error, we find that the mass of flow field is increased with the MGFM, while it is decreased with the RGFM. In Fig. 3(2), 3(3) and 3(4), the cases for the MGFM and $\alpha=1$ is not shown since the conservation error now is very large and the procedure breaks down after a few time steps of computation. Indeed, for two-medium flow with large density ratio and large pressure ratio across the interface, the numerical solutions close to the interface is less accuracy due to the overheating problem, which indicates that the input states of Riemann problem should be far from the interface $(\alpha=2)$ for the MGFM with stronger contact discontinuity. However, the value of $\alpha$ has nearly no differences for the RGFM. The conservation error measured with the RGFM tends to be flat with increased density ratio for all the cases, while it tends to be divergent for the MGFM under larger pressure ratio. Except for $p_{L} / p_{R}=10$, the conservation error computed with the RGFM is smaller than the MGFM. Therefore we can conclude that for gas-gas sod tube, when $p_{L} / p_{R}$ is small, the numerical results obtained with the MGFM has better performances, while the RGFM is recommended for large pressure ratio across the interface.

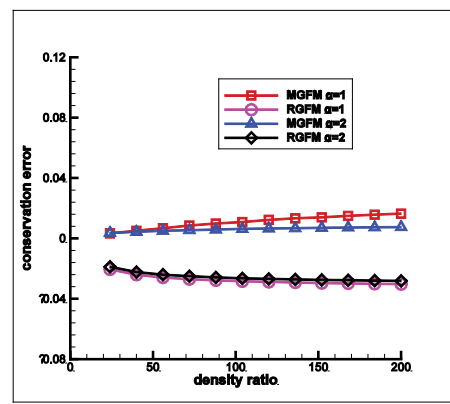

(1) $p_{L} / p_{R}=10$ at $t=0.15$.

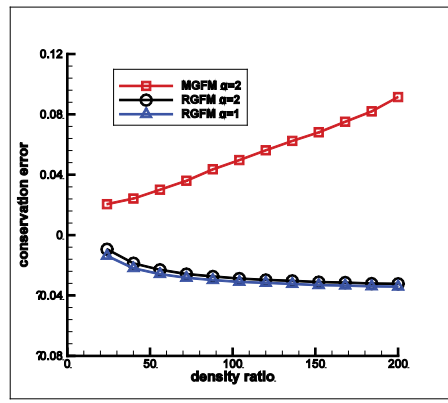

(3) $p_{L} / p_{R}=1000$ at $t=0.03$.

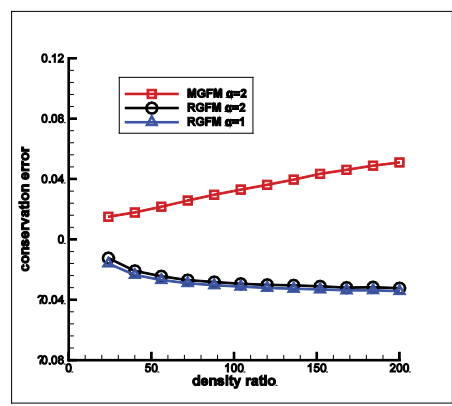

(2) $p_{L} / p_{R}=100$ at $t=0.12$.

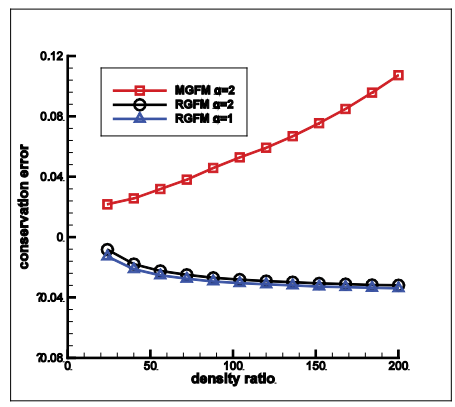

(4) $p_{L} / p_{R}=10000$ at $t=0.01$

Fig. 3. The conservation error for gas-gas sod tube.

\subsubsection{Gas-water sod tube}

We then change the medium 2 into water $(\gamma=7.15, B=3309)$ and set $\rho_{R}=1.0, p_{R}=1.0$ as constant in Eq. (25) to investigate the effects by different GFMs for gas-water problem. 
The density ratio and pressure ratio is: $\rho_{L} / \rho_{R}=0.002,0.01,0.5,2,5,10,20, p_{L} / p_{R}=100$, $1000,10000,100000$. The conservation error is shown in table 1 . We can find that the mass of flow field is always decreased with increased density ratio. With the MGFM, $\alpha=2$ should be used with large density ratio and low pressure ratio, however $\alpha=1$ is recommended for other cases. Similar to the gas-gas sod tube, the conservation error is nearly not dependent on the value of $\alpha$ for the RGFM. In general, both GFMs have the same effects on the conservation error for weak contact discontinuity. However, the MGFM is more robust and suitable, especially for the cases with large density ratio and large pressure ratio. Thus the MGFM is suggested for solving the gas-water sod tube.

Table 1. The conservation error $\left(\times 10^{-4}\right)$ for gas-water sod tube.

(1) $p_{L} / p_{R}=100$ at $t=0.0028$.

\begin{tabular}{cccccccc}
\hline$\rho_{L} / \rho_{R}$ & 0.002 & 0.01 & 0.5 & 2 & 5 & 10 & 20 \\
\hline MGFM $\alpha=2$ & 6 & 6 & 1.5 & -1 & -4 & -5 & -6 \\
MGFM $\alpha=1$ & 4 & 4 & 1.3 & -2 & -8 & -9 & -11.5 \\
RGFM $\alpha=2$ & 6 & 5 & 0.8 & -10 & -29 & -48 & -68 \\
RGFM $\alpha=1$ & 8 & 7 & 1 & -12 & -30 & -52 & -73 \\
\hline
\end{tabular}

(2) $p_{L} / p_{R}=1000$ at $t=0.0025$.

\begin{tabular}{cccccccc}
\hline$\rho_{L} / \rho_{R}$ & 0.002 & 0.01 & 0.5 & 2 & 5 & 10 & 20 \\
\hline MGFM $\alpha=2$ & 7.2 & 7.1 & 6 & 1.5 & -1.1 & -1.2 & -1.3 \\
$\operatorname{MGFM} \alpha=1$ & 6.9 & 6.9 & 5 & 1.2 & -2.2 & -2.4 & -2.5 \\
RGFM $\alpha=2$ & 11 & 10 & 8 & -20 & -55 & -90 & -130 \\
RGFM $\alpha=1$ & 10 & 9 & 4 & -22 & -60 & -96 & -140 \\
\hline
\end{tabular}

(3) $p_{L} / p_{R}=10000$ at $t=0.0015$.

\begin{tabular}{cccccccc}
\hline$\rho_{L} / \rho_{R}$ & 0.002 & 0.01 & 0.5 & 2 & 5 & 10 & 20 \\
\hline MGFM $\alpha=2$ & 22 & 21 & 13 & 10 & 8.5 & 7 & 6.9 \\
MGFM $\alpha=1$ & 13 & 12 & 7.3 & 5 & 3.7 & 3.2 & 3.0 \\
RGFM $\alpha=2$ & 35 & 34 & 25 & -17 & -75 & -130 & -175 \\
RGFM $\alpha=1$ & 30 & 29 & 19 & -24 & -83 & -135 & -190 \\
\hline
\end{tabular}

(4) $p_{L} / p_{R}=100000$ at $t=0.0005$.

\begin{tabular}{cccccccc}
\hline$\rho_{L} / \rho_{R}$ & 0.002 & 0.01 & 0.5 & 2 & 5 & 10 & 20 \\
\hline MGFM $\alpha=2$ & 36 & 35 & 30 & 19 & 15 & 15 & 12 \\
MGFM $\alpha=1$ & 26 & 26 & 22 & 12 & 7 & 6 & 4 \\
RGFM $\alpha=2$ & 53 & 52 & 45 & -12 & -78 & -140 & -195 \\
RGFM $\alpha=1$ & 50 & 50 & 36 & -18 & -86 & -155 & -210 \\
\hline
\end{tabular}




\subsection{Shock-bubble interaction}

Shock wave interactions with bubbles have been studied experimentally by Hass and Sturtevant $^{30}$, and Takayama ${ }^{31}$. It also has been numerically investigated by several authors, see Refs 32-35 for details. Here we investigate the effects with different GFMs for the shock impacting on the bubble problem. Fig. 4 shows the computational set-up and the geometrical parameters are: $a=1, \mathrm{~b}=0.5, c=2, d=6.5, e=0.89$. A shock wave propagates to the left and hits a helium bubble placed within the air. On the left and right boundaries, nonreflecting boundary condition is used and the upper and lower boundaries are treated as slip-wall. The computational domain is divided into $650 \times 178$ mesh cells and the TVB limiter constant is taken as $M=0.1$. The non-dimensional initial conditions are: $\rho=1, u=0, v=0, p=1 / 1.4, \gamma=1.4, B=0$ for pre-shocked air, $\rho=0.1819, u=0$, $v=0, p=1 / 1.4, \gamma=1.648, B=0$ for helium. The shock strength is set as: $p_{s}=3,4.5,6$, $7.5,9,10.5,12,13.5,15,16.5,18,19.5,21,22.5,24,25.5,27,28.5$ and 30 , respectively.

At the initial stage, the interface swept over by the shock wave is set into motion while the others remain at rest. During the contraction of the bubble, the length of the interface decreases and then the interface begins to fold down over itself. The ambient fluid piercing the bubble grows, thus an overpressure behind the bubble is formed to compress the bubble and move it to the left part of the computational domain. The shock wave diffracts into two waves after it hits the helium bubble. Part of the shock wave is transmitted to the bubble with a stronger intensity than that of the incident shock along the normal direction of the interface. With increased shock strength, the bubble is contracted more intensely and is set into motion in a faster way. Under a series of shock strength $p_{s}$, Fig. 5 shows the conservation errors as defined in Eq. (26) where now $M_{n}$ is the mass of helium bubble at time $t^{n}$ and $M_{0}$ is the initial mass of helium bubble. It is found that after the shock impacts on the bubble, the mass of helium bubble is increased with the passage of time. Meanwhile, the conservation error is larger with stronger shock strength. The RGFM with $\alpha=1$ and the MGFM with $\alpha=2$ give similar results, while the RGFM with $\alpha=2$ presents the worst performances. The conservation error with $\alpha=2$ is larger in all the cases, which indicates that the input states of Riemann problem should be close to the interface $(\alpha=1)$ in this test. The conservation error caused with the MGFM is always smaller than the RGFM, and therefore the MGFM is more suitable for the problem with shock impacting on the curved gas-gas interface.

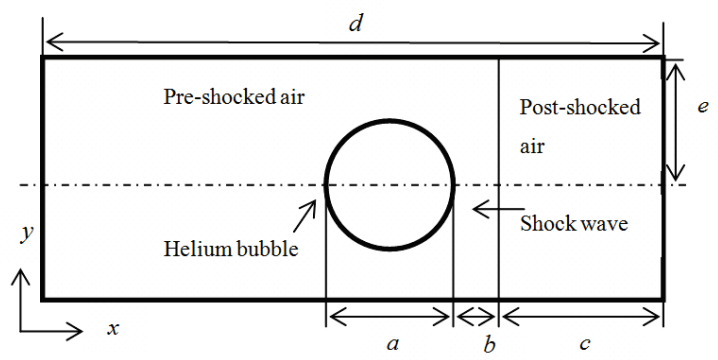

Fig. 4. A schematic of computational domain (not to scale). 


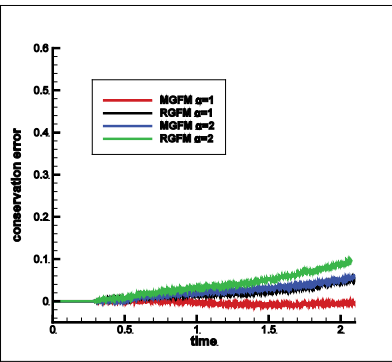

(1) $p_{s}=3$.

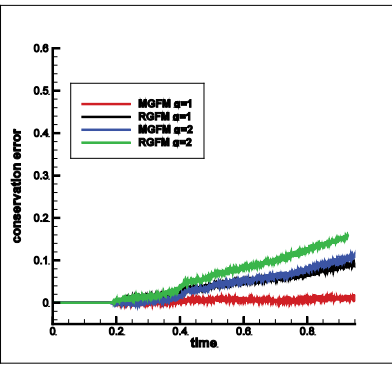

(4) $p_{s}=7.5$.

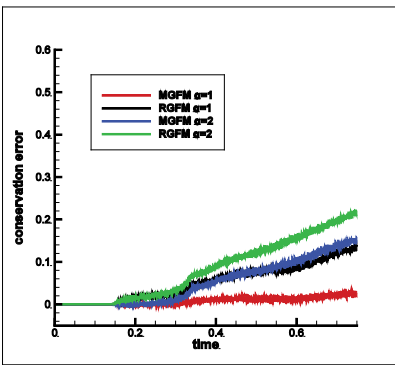

(7) $p_{s}=12$.

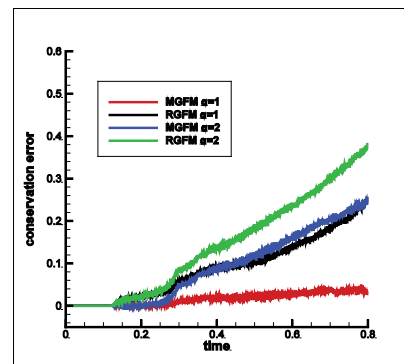

(10) $p_{s}=16.5$.

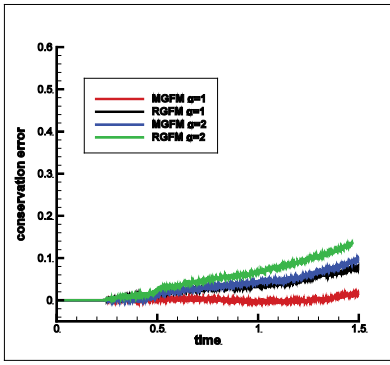

(2) $p_{s}=4.5$.

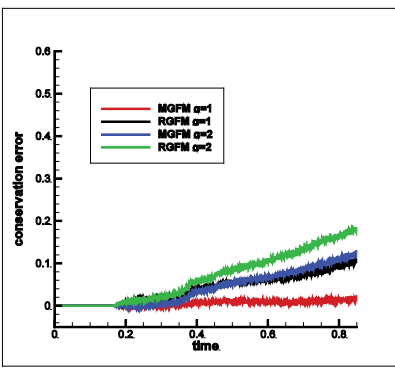

(5) $p_{s}=9$.

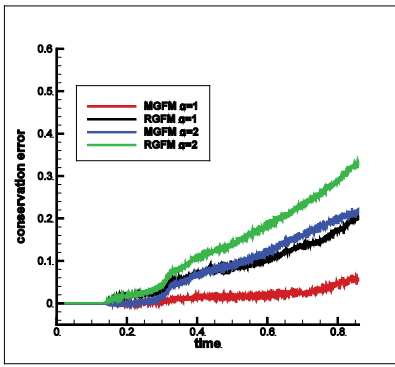

(8) $p_{s}=13.5$.

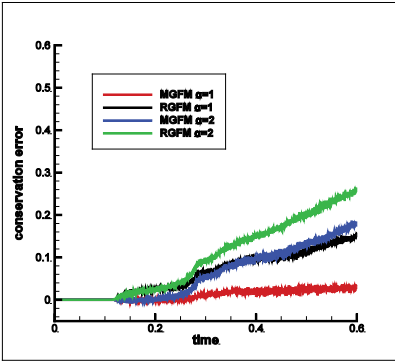

(11) $p_{s}=18$.

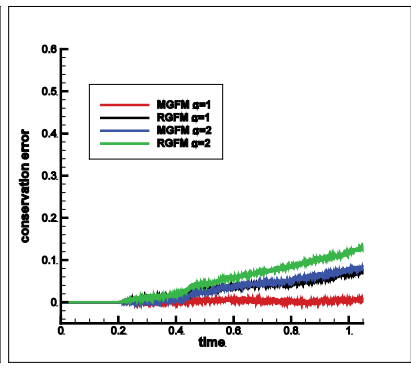

(3) $p_{s}=6$.

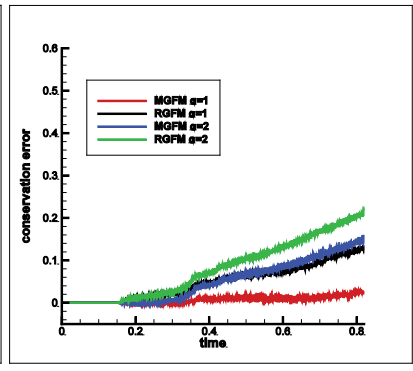

(6) $p_{s}=10.5$.

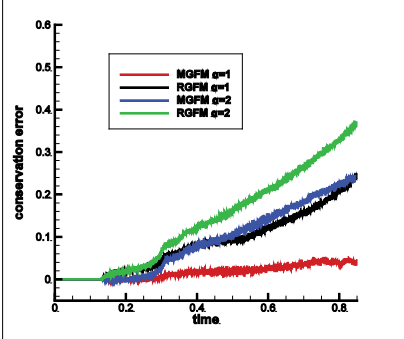

(9) $p_{s}=15$.

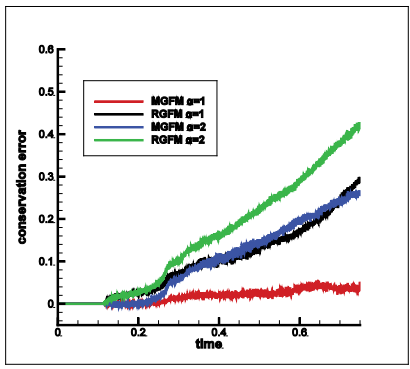

(12) $p_{s}=19.5$. 


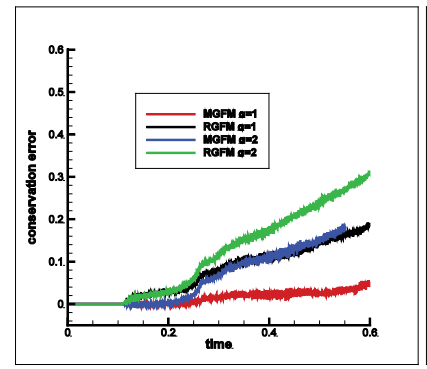

(13) $p_{s}=21$.

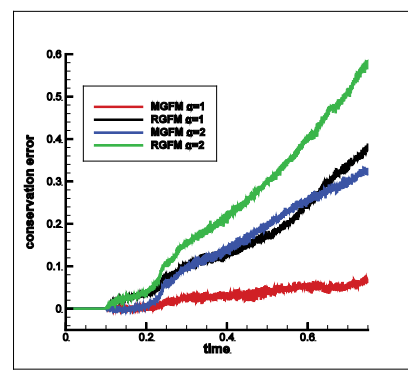

(16) $p_{s}=25.5$.

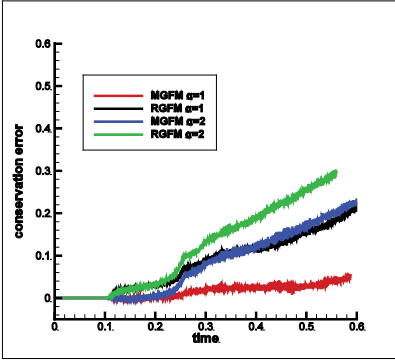

(14) $p_{s}=22.5$.

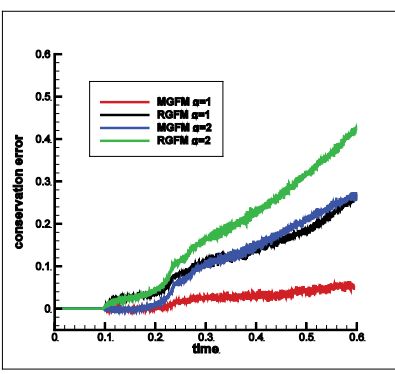

(17) $p_{s}=27$.

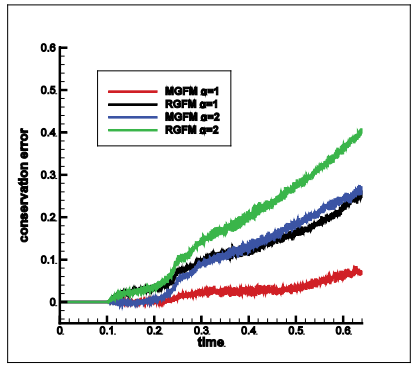

(15) $p_{s}=24$.

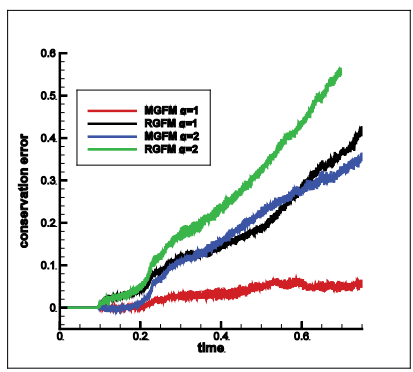

(18) $p_{s}=28.5$.

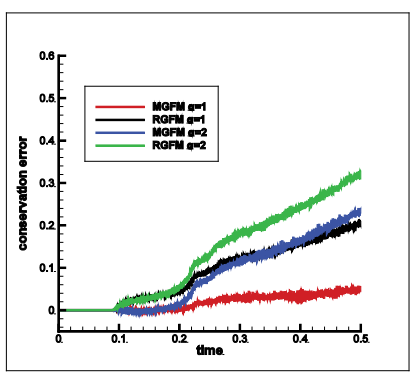

(19) $p_{s}=30$.

Fig. 5. The conservation error for the shock-bubble interaction.

\section{Concluding remarks}

In this paper, the different effects of MGFM and RGFM on the compressible twomedium flow in two dimensions are investigated. The motion of the interface is obtained by constructing the Riemann problem nearby the interfacial region and the Euler equations are solved with the RKDG method. Extensive numerical tests, including the sod tube and the shock-bubble interaction, are tested to obtain the optimum matching of the GFMs under various initial conditions of flow field. The gas-gas sod tube study shows that when the pressure ratio is small, the MGFM has better performances, while the RGFM is recommended for large pressure ratio across the interface. The numerical results of gas-water sod tube indicate that the MGFM is more suitable for all the cases in 
general. In simulations of shock-bubble interaction, it demonstrates that the MGFM is stable and gives smaller conservation error when we increase the shock strength. We hope that these conclusions can provide an empirical guidance for simulating the compressible two-medium flow. Finally, it must be emphasized that the conclusions from these numerical tests are obtained from the perspective of conservation error, other indicators, for example, the geometrical information of the interface can also be used to investigate the effects with various GFMs, which will be reported elsewhere.

\section{Acknowledgments}

All the authors are supported by NSFC grants 91130030 and 11432007. Additional support is provided by a project funded by the Priority Academic Program Development (PAPD) of Jiangsu Higher Education Institutions.

\section{References}

1. M. Brouillette, The Richtmyer-Meshkov instability, Annu. Rev. Fluid Mech., 34(2002), 445468.OPAL Collab. (G. Abbiendi et al.), Eur. J. Phys. C 11, 217 (1999).

2. G. Tryggvason and S.O. Unverdi, Computations of three-dimensional Rayleigh-Taylor instability, Phys. Fluids A, 2 (1990), 656-659.

3. W.H. Reed and T. R. Hill, Triangular Mesh Methods for the Neutron Transport Equation, Los Alamos Scientific Laboratory Report LA-UR-73-479, 1973.

4. B. Cockburn, S. Hou and C.-W. Shu, The Runge-Kutta local projection discontinuous Galerkin finite element method for conservation laws IV: the multidimensional case, Math. Comput., 54(1990), 545-581.

5. B. Cockburn, S.-Y. Lin and C.-W. Shu, TVB Runge-Kutta local projection discontinuous Galerkin finite element method for conservation laws III: one dimensional systems, J. Comput. Phys., 84 (1989), 90-113.

6. B. Cockburn and C.-W. Shu, TVB Runge-Kutta local projection discontinuous Galerkin finite element method for conservation laws II: general framework, Math. Comput., 52 (1989), 411435.

7. B. Cockburn and C.-W. Shu, The Runge-Kutta local projection P1-discontinuous Galerkin finite element method for scalar conservation laws, Math. Model. Numer. Anal., 25 (1991), 337-361.

8. B. Cockburn and C.-W. Shu, The Runge-Kutta discontinuous Galerkin method for conservation laws V: multidimensional systems, J. Comput. Phys., 141 (1998), 199-224.

9. C.-W. Shu, S. Osher, Efficient implementation of essentially non-oscillatory shock capturing schemes, J. Comput. Phys., 77 (1988), 439-471.

10. C.-W. Shu, TVB uniformly high-order schemes for conservation laws, Math. Comp., 49 (1987), 105-121.

11. S. Karni, Multicomponent flow calculations by a consistent primitive algorithm, J. Comput. phys., 112 (1994), 31-43.

12. R. Abgrall, How to prevent pressure oscillations in multicomponent flow calculations: a quasi conservative approach, J. Comput. Phys., 125 (1996), 150-160.

13. B. Larrouturou, How to preserve the mass fractions positivity when computing compressible multi-component flows, J. Comput. Phys., 95 (1991), 59-84.

14. W. Mulder, S. Osher and J.A. Sethian, Computing interface motion in compressible gas dynamics, J. Comput. Phys, 100 (1992), 209-228. 
15. S. Osher and R.P. Fedkiw, Level set methods: An overview and some recent results, J. Comput. Phys., 169 (2001), 463-502.

16. I.-L. Chern, J. Glimm, O. McBryan, B. Plohr and S. Yaniv, Front tracking for gas dynamics, J. Comput. Phys., 62 (1986), 83-110.

17. J. Glimm, J.W. Grove, X.L. Li, K.-M Shyue, Y. Zeng and Q. Zhang, Three-dimensional front tracking, SIAM J. Sci. Comput., 19 (1998), 703-727.

18. J. Glimm, J.W. Grove, X.L. Li and N. Zhao, Simple front tracking, Contemp. Math., 238 (1999), 133-149.

19. J. Glimm, J. W. Grove, X.L. Li, W. Oh and D.H. Sharp, A critical analysis of Rayleigh-Taylor growth rates, J. Comput. Phys., 169 (2001), 652-677.

20. R. Caiden, R.P. Fedkiw and C. Anderson, A numerical method for two-phase flow consisting of separate compressible and incompressible regions, J. Comput. Phys., 166 (2001), 1-27.

21. R.P. Fedkiw, Coupling an Eulerian fluid calculation to a Lagrangian solid calculation with the ghost fluid method, J. Comput. Phys., 175 (2002), 200-224.

22. R.P. Fedkiw, T. Aslam, B. Merriman and S. Osher, A non-oscillatory Eulerian approach to interfaces in multimaterial flows (the ghost fluid method), J. Comput. Phys., 152 (1999), 457492.

23. X.Y. Hu and B.C. Khoo, An interface interaction method for compressible multifluids, J. Comput. Phys., 198 (2004), 35-64.

24. T.G. Liu, B.C. Khoo and K.S. Yeo, Ghost fluid method for strong shock impacting on material interface, J. Comput. Phys., 190 (2003), 651-681.

25. T.G. Liu, B.C. Khoo and C.W. Wang, The ghost fluid method for compressible gas-water simulation, J. Comput. Phys., 204 (2005), 193-221.

26. C.W. Wang, T.G. Liu and B.C. Khoo, A real ghost fluid method for the simulation of multimedium compressible flow, SIAM J. Sci. Comput., 28 (2006), 278-302.

27. R. Saurel and R. Abgrall, A simple method for compressible multifluid flows, SIAM J. Sci. Comput., 21 (1999), 1115-1145.

28. T.G. Liu, B.C. Khoo and K.S. Yeo, The simulation of compressible multi-medium flow. I. A new methodology with test applications to 1D gas-gas and gas-water cases, Comput. \& Fluids, 30 (2001), 291-314.

29. J.-P. Cocchi and R. Saurel, A Riemann problem based method for the resolution of compressible multimaterial flows, J. Comput. Phys., 137 (1997), 265-298.

30. J.-F. Hass and B. Sturtevant, Interaction of weak shock waves with cylindrical and spherical gas inhomogeneities, J. Fluid Mech., 181 (1987), 41-76.

31. K. Takayama, Holographic interferometric study of shock wave propagation in two-phase media, in: Shock Tubes and Waves, Proc 16th Intl. Symp. On Tubes and Waves, Aachen, 5162, 1987.

32. R.R. Nourgaliev, T.N. Dinh and T.G. Theofanous, Adaptive characteristics-based matching for compressible multifluid dynamics, J. Comput. Phys., 213 (2006), 500-529.

33. J.M. Picone and J.P. Boris, Vorticity generation by shock propagation through bubbles in a gas, J. Fluid Mech., 189(1988), 23-51.

34. J.J. Quirk and S. Karni, On the dynamics of a Shock-bubble interaction, J. Fluid Mech., 318(1996), 129-163.

35. H. Terashima and G. Tryggvason, A front tracking/ghost fluid method for fluid interfaces in compressible flows, J. Comput. Phys., 228 (2009), 4012-4037. 